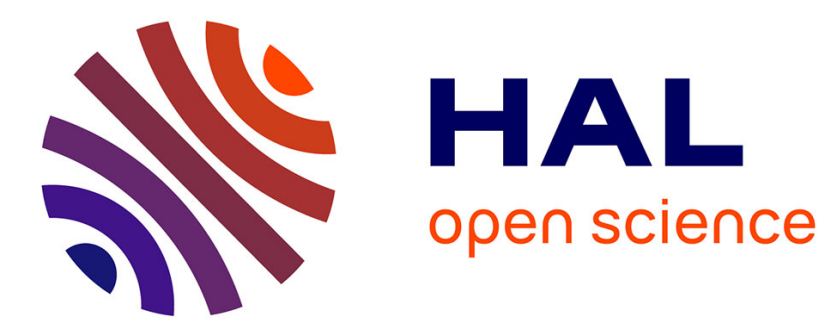

\title{
Measurements of quarkonium production with ALICE at the LHC
}

Javier Castillo

\section{To cite this version:}

Javier Castillo. Measurements of quarkonium production with ALICE at the LHC. Journal of Physics G: Nuclear and Particle Physics, 2010, 37 (9), pp.94051. 10.1088/0954-3899/37/9/094051 . hal00600858

\section{HAL Id: hal-00600858 https://hal.science/hal-00600858}

Submitted on 16 Jun 2011

HAL is a multi-disciplinary open access archive for the deposit and dissemination of scientific research documents, whether they are published or not. The documents may come from teaching and research institutions in France or abroad, or from public or private research centers.
L'archive ouverte pluridisciplinaire HAL, est destinée au dépôt et à la diffusion de documents scientifiques de niveau recherche, publiés ou non, émanant des établissements d'enseignement et de recherche français ou étrangers, des laboratoires publics ou privés. 


\title{
Measurements of quarkonium production with ALICE at the LHC
}

\author{
Javier Castillo for the ALICE Collaboration \\ CEA, Centre de Saclay, IRFU/SPhN, 91191 Gif-sur-Yvette, France \\ E-mail: jcastill@cea.fr
}

\begin{abstract}
ALICE is the LHC experiment dedicated to the study of heavy-ion collisions. The main purpose of ALICE is to investigate the properties of a new state of deconfined nuclear matter, the quark gluon plasma. Quarkonium measurements are very promising tools to unveil the properties of the quark gluon plasma. We will review the capabilities of the ALICE detectors to measure heavy quarkonia at both mid and forward-rapidity regions, and discuss the related physics programme.
\end{abstract}

\section{Introduction}

In ultra-relativistic heavy-ion collisions, we aim to investigate the properties of nuclear matter under extreme conditions of temperature and pressure which are expected to lead to the creation of deconfined partonic matter, the Quark Gluon Plasma (QGP). With the objective of studying the QGP, heavy-ion collisions were studied at the CERN SPS and are currently under investigation at RHIC at BNL. The Large Hadron Collider (LHC) at CERN, which delivered its first proton-proton ( $\mathrm{p}-\mathrm{p}$ ) collisions in November 2009, will collide Lead to Lead $(\mathrm{Pb}-\mathrm{Pb})$ ions at $\sqrt{\mathrm{S}_{\mathrm{NN}}}=5.5 \mathrm{TeV}$ providing so far unprecedented conditions to study the QGP.

While several observables have been proposed to characterize the QGP, the study of heavy quark ( $c$ and $b$ ) production is thought to be one of the most powerful probes. The study of the production of heavy quark and anti-quark bound states (quarkonia), $\mathrm{J} / \psi$, $\psi^{\prime}(c \bar{c}), \Upsilon(1 \mathrm{~S}), \Upsilon(2 \mathrm{~S})$ and $\Upsilon(3 \mathrm{~S})(b \bar{b})$ is particularly interesting. It was first proposed that quarkonium resonances will dissociate by color screening in the QGP [1], thus a suppression of quarkonium production in nucleus-nucleus collisions compared to protonnucleus collisions was predicted as a signature of the QGP formation. Later, it was also proposed that additional quarkonium production mechanisms, such as statistical hadronization [2] or quark and anti-quark recombination in the QGP [3], could add to the prompt production by initial hard scattering. In this case an enhanced production of quarkonium resonances will be observed in nucleus-nucleus collisions. The regeneration scenarios are expected to be important for the charmonium states at LHC energies. 
Figure 1. General layout of the ALICE detector.

\section{The ALICE experiment at the LHC}

ALICE is a general-purpose heavy-ion experiment designed to study the physics of strongly interacting matter and the QGP in nucleus-nucleus collisions at the LHC. The detector is designed to cope with the highest particle multiplicities anticipated for $\mathrm{Pb}-$ $\mathrm{Pb}$ collisions $\left(d N_{c h} / d y\right.$ up to 8000$)$ and was operational for the first p-p collisions at the LHC in November 2009. In addition to heavy systems, ALICE will study collisions of lower-mass ions and protons (both $\mathrm{p}-\mathrm{p}$ and $\mathrm{p}-\mathrm{A}$ ), which primarily provide reference data for the nucleus-nucleus collisions. This data will allow for a number of genuine $\mathrm{p}-\mathrm{p}$ and p-A physics studies. The first p-p physics result was produced just one week after the first ever LHC collision [4].

The detector (Fig. 1) consists of a central part, which measures hadrons, electrons and photons, and of a forward spectrometer to measure muons. The central part, which covers polar angles from $45^{\circ}$ to $135^{\circ}$ over the full azimuth, is embedded in the L3 solenoidal magnet. It consists of various tracking detectors and particle identification arrays among which are the Inner Tracking System (ITS), a cylindrical Time-Projection Chamber (TPC) and a Transition Radiation Detector (TRD). The forward muon arm covers polar angles from $171^{\circ}$ to $178^{\circ}$. Several smaller detectors (ZDC, FMD, V0...) for global event characterization and triggering are located at forward-backward angles. More details can be found in [5].

\section{Quarkonia detection capabilities}

ALICE has good capabilities to measure quarkonium production in two rapidity domains, at mid rapidity through their dielectron decay and at forward rapidity via their dimuon decay.

\subsection{At mid rapidity in the dielectron channel}

Electron pairs will be measured in the ALICE central barrel in the $|y|<0.9$ rapidity domain. Electrons are tracked and identified by the following detectors:

- ITS [5] allows track finding, primary vertex reconstruction and secondary vertex finding. It is composed of three subsystems of two layers each: the Silicon Pixel Detector, the Silicon Drift Detector and the Silicon Strip Detector.

- TPC [6, 5], optimized for large multiplicity, allows track finding, momentum measurement, and charged hadron and low momentum electron identification via $d E / d x$. The track momentum resolution, including TPC and ITS information, is expected to be better than $2 \%$ for transverse momentum $p_{\perp}<20 \mathrm{GeV} / \mathrm{c}$.

- TRD [5] allows electron identification for momenta larger than $1 \mathrm{GeV} / \mathrm{c}$. 
Table 1. Expected mass resolution $\left(\sigma_{m}\right)$, signal rate (S), signal-to-background ratio and significance for $\mathrm{J} / \psi$ and $\Upsilon$ in the dielectron channel within $\pm 1.5 \sigma$ around each resonance mass for $10 \%$ most central $\mathrm{Pb}-\mathrm{Pb}$ collisions. All yields correspond to a nominal year (see text) of data taking.

\begin{tabular}{ccccc}
\hline state & $\sigma_{m}(\mathrm{MeV})$ & $\mathrm{S}\left(\mathrm{x} 10^{3}\right)$ & $\mathrm{S} / \mathrm{B}$ & $\mathrm{S} / \sqrt{S+B}$ \\
\hline $\mathrm{J} / \psi$ & 33 & 121.1 & 1.4 & 265 \\
$\Upsilon$ & 90 & 1.3 & 1.6 & 21 \\
\hline
\end{tabular}

Table 2. Expected mass resolution $\left(\sigma_{m}\right)$, signal rate $(\mathrm{S})$, signal-to-background ratio and significance for $\mathrm{J} / \psi$ and $\Upsilon$ in the dimuon channel with an interval of $\pm 2.0 \sigma$ around each resonance mass for minimum-bias $\mathrm{Pb}-\mathrm{Pb}$ collisions. All yields correspond to a nominal year (see text) of data-taking.

\begin{tabular}{ccccc}
\hline state & $\sigma_{m}(\mathrm{MeV})$ & $\mathrm{S}\left(\mathrm{x} 10^{3}\right)$ & $\mathrm{S} / \mathrm{B}$ & $\mathrm{S} / \sqrt{S+B}$ \\
\hline $\mathrm{J} / \psi$ & 70 & 676.7 & 0.3 & 410 \\
$\Upsilon$ & 100 & 6.8 & 2.7 & 71 \\
\hline
\end{tabular}

The invariant mass resolution for the quarkonia was studied using detailed GEANT simulations. The reconstructed peaks were fitted by a Gaussian and the invariant mass resolution for the $\mathrm{J} / \psi$ and $\Upsilon$ were found to be $\sigma_{m}^{\mathrm{J} / \psi}=33 \mathrm{MeV} / \mathrm{c}^{2}$ and $\sigma_{m}^{\Upsilon}=90 \mathrm{MeV} / \mathrm{c}^{2}$ respectively. The expected number of $\mathrm{J} / \psi$ and $\Upsilon$ to be reconstructed by the central barrel in a nominal year and for $\mathrm{dN}_{c h} / \mathrm{d} y=3000$ are summarized in Table 1 . A nominal year is defined by a $10^{6} \mathrm{~s}$ data taking period of $\mathrm{Pb}-\mathrm{Pb}$ collisions at a luminosity of $5 \times 10^{26} \mathrm{~cm}^{-2} \mathrm{~s}^{-1}\left(10^{7} \mathrm{~s}\right.$ at a luminosity of $5 \times 10^{30} \mathrm{~cm}^{-2} \mathrm{~s}^{-1}$ for $\mathrm{p}$-p collisions $)$. Our simulations showed that the $\mathrm{J} / \psi$ can be reconstructed up to $p_{\perp} \sim 10 \mathrm{GeV} / \mathrm{c}$.

\subsection{At forward rapidity in the dimuon channel}

Muon pairs will be reconstructed by the ALICE forward muon spectrometer [5] in an acceptance region of $-4<y<-2.5$ and with full azimuthal coverage. The spectrometer consists of

- a front absorber to stop most hadrons, electrons and photons coming from the interaction point,

- an inner beam shield to stop re-scattered particles from the beam pipe,

- 10 tracking planes to allow particle trajectory reconstruction,

- a large area warm 3.0 Tm dipole magnet for momentum determination,

- and a passive muon filter wall followed by 4 trigger planes that will provide single muon and muon pair triggers.

We have also used full simulations to study the muon spectrometer performances for the quarkonium reconstruction. We found the invariant mass resolution for the $\mathrm{J} / \psi$ and $\Upsilon$ to be $\sigma_{m}^{\mathrm{J} / \psi}=70 \mathrm{MeV} / \mathrm{c}^{2}$ and $\sigma_{m}^{\Upsilon}=100 \mathrm{MeV} / \mathrm{c}^{2}$ respectively. 
The expected numbers of $\mathrm{J} / \psi$ and $\Upsilon$ to be reconstructed by ALICE in the muon spectrometer in a nominal year are summarized in Table 2 . It is worth noting that the statistics for a nominal year will enable detailed differential studies of $\mathrm{J} / \psi$ production in $\mathrm{Pb}-\mathrm{Pb}$ collisions. We expect to be able to reconstruct the $\mathrm{J} / \psi$ from $p_{\perp}=0 \mathrm{GeV} / \mathrm{c}$ up to $p_{\perp} \sim 20 \mathrm{GeV} / \mathrm{c}$. It is also important to note that the expected mass resolution and statistics are sufficient to separate the three states of the $\Upsilon$ family.

For details on the ALICE capabilities to measure quarkonia production at both mid and forward-rapidity and on the expected rates in both $\mathrm{p}-\mathrm{p}$ and $\mathrm{Pb}-\mathrm{Pb}$ collisions see [7].

\section{Quarkonium observables}

Quarkonium measurements in p-p collisions are an important part of the ALICE physics program. They will allow to constrain the quarkonium production models and will establish the baseline for the nucleus-nucleus collisions. In this contribution we will however focus on quarkonium measurements in heavy-ion collisions.

The bread and butter of the quarkonium production studies are the $\mathrm{J} / \psi$ suppression, or enhancement, measurements. Our simulations showed that in $\mathrm{Pb}-\mathrm{Pb}$ collisions ALICE can perform them in two rapidity domains $(|y|<0.9$ and $-4<y<-2.5)$ differentially in at least five centrality classes and as a function of $p_{\perp}[7]$.

\subsection{Normalizations for charmonium studies}

For the $\mathrm{J} / \psi$ yields normalization in $\mathrm{Pb}-\mathrm{Pb}$ collisions several options are being investigated. First, we can measure the $R_{C P}$, i.e., the $\mathrm{J} / \psi$ yields in central collisions compared to the most peripheral collisions, respectively normalized by their number of binary collisions $\left(N_{\text {coll }}\right)$. The second option is a $R_{A A}$ measurement, i.e., the yields in $\mathrm{Pb}-\mathrm{Pb}$ collisions compared to those in $\mathrm{p}-\mathrm{p}$ collisions normalized by $N_{\text {coll }}$. Since the reference for $R_{A A}$, p-p collisions, is cleaner than the one for $R_{C P}, R_{A A}$ is expected to be more educating than $R_{C P}$. However, it has the drawback that p-p collisions will be initially measured at a higher centre of mass energy. Third, we can normalize with respect to beauty production, which can be measured from semi-leptonic decays at both, mid and forward-rapidity [7]. In the muon spectrometer beauty production can also be measured from the low and high-mass dimuons originating from correlated $\mathrm{B} \overline{\mathrm{B}}$ pair decays [7]. Finally, the most meaningful normalization to the $\mathrm{J} / \psi$ cross-section, if the regeneration mechanism dominates, will be the charm cross-section. The charm cross-section can be measured in ALICE at mid-rapidity [7].

\subsection{Secondary $\mathrm{J} / \psi$ production}

A good knowledge of the sources of secondary $\mathrm{J} / \psi$ is crucial for the interpretation of the suppression results. This knowledge is poor. At low energy and for p-p collisions, the contribution to the produced $\mathrm{J} / \psi$ from the decay of the excited states $\psi^{\prime}$ and 
Figure 2. $\Upsilon^{\prime} / \Upsilon$ ratio as a function of the collision centrality for a no suppression scenario (closed circles), one with high deconfinement temperature $\mathrm{T}_{d}=270 \mathrm{MeV}$ (closed triangles) and one with $\mathrm{T}_{d}=190 \mathrm{MeV}$ (open circles) [7]. See text for details.

$\chi_{c}$ was recently evaluated to be $(8.1 \pm 0.3) \%$ and $(25 \pm 5) \%$, respectively [8]. The currently available data at higher energy does not allow to determine an eventual energy dependence of the above feed-down. Also, for nucleus-nucleus collisions the feed-down from excited states might differ. At the LHC the situation will be more complicated. Indeed, in addition to the above ones, the contribution from $\mathrm{B}$ hadron decays will also be important. At the LHC this contribution is expected to be $\sim 20 \%$. ALICE can measure the $\mathrm{J} / \psi$ from $\mathrm{B}$ decays in the central barrel by measuring the displaced secondary vertex. A technique to statistically measure $\mathrm{J} / \psi$ from $\mathrm{B}$ decays in the muon spectrometer, based on $\mathrm{J} / \psi$-muon correlations, is also under investigation. The number of $\mathrm{J} / \psi$ originating from $\psi^{\prime}$ decays will be estimated from the direct $\psi^{\prime}$ yield measured in the dimuon channel. At mid-rapidity, a technique to measure the secondary $\mathrm{J} / \psi$ coming from the radiative $\chi_{c}$ decays is under study [9]. The $\mathrm{J} / \psi$ is measured in its dielectron channel thanks to the ITS, TPC and TRD while the photon is measured by conversion with the $e^{+}$and $e^{-}$being measured in the TPC.

\subsection{Quarkonium polarization}

Quarkonium polarization measurements should allow to distinguish different production mechanisms, since different models predict different polarizations. Quarkonium polarization can be reconstructed from the angular distribution of the decay products (dimuons or dielectrons). It has been predicted that an increase of $\mathrm{J} / \psi$ polarization may be expected if the QGP is formed [10]. Our simulations have shown that for both $\mathrm{p}$ - $\mathrm{p}$ and $\mathrm{Pb}-\mathrm{Pb}$ collisions differential measurements of $\mathrm{J} / \psi$ polarization versus $p_{\perp}$ and centrality will be possible after a nominal year of data taking while for the $\Upsilon$ polarization integrated measurements will be performed.

\section{4. $\Upsilon$ measurements}

The high luminosity and energy conditions at the LHC will enable the measurements of the $\Upsilon$ family. While several $\Upsilon$ normalizations will be studied [11], the $\Upsilon^{\prime}$ over $\Upsilon$ ratio is expected to be particularly sensitive to the QGP suppression mechanism. Figure 2 shows the expected $\Upsilon^{\prime} / \Upsilon$ ratio as a function of the collision centrality for three different QGP suppression scenarii, one (closed triangles) characterized by a high deconfinement temperature $\mathrm{T}_{d}=270 \mathrm{MeV}$ [12], another one (open circles) using $\mathrm{T}_{d}=190 \mathrm{MeV}$ [13] and a no suppression scenario (closed circles). The statistics correspond to a nominal year of data taking as defined previously. We observe that the $\Upsilon^{\prime} / \Upsilon$ has a good discriminating power between the different suppression scenarii. 


\section{Conclusion}

We summarized the expected performances of ALICE to investigate quarkonium physics. We showed that ALICE has good potential to contribute to the understanding of quarkonium physics in heavy-ion collisions and to help investigate the properties of the quark gluon plasma. The ALICE experiment has successfully entered the data-taking era and the detector is ready to continue.

\section{References}

[1] T. Matsui and H. Satz, Phys. Lett. B 178 (1986) 416.

[2] P. Braun-Munzinger and J. Stachel, Phys. Lett. B 490 (2000) 196.

[3] R. L. Thews, M. Schroedter and J. Rafelski, Phys. Rev. C 63 (2001) 054905.

[4] K. Aamodt et al. (ALICE Collaboration), Eur. Phys. J. C 65 (2010) 111.

[5] K. Aamodt et al. (ALICE Collaboration), JINST 0803 (2008) S08002 [JINST 3 (2008) S08002].

[6] J. Alme et al., arXiv:1001.1950 [physics.ins-det], submitted to Nucl. Instr. Meth.

[7] B. Alessandro et al. (ALICE Collaboration), J. Phys. G 32 (2006) 1295.

[8] P. Faccioli, C. Lourenco, J. Seixas and H. K. Woehri, JHEP 0810 (2008) 004.

[9] P. Gonzalez et al. for the ALICE Collaboration, Eur. Phys. J. C 61 (2009) 899 [Erratum-ibid. C 61 (2009) 915].

[10] B. L. Ioffe, D. E. Kharzeev, Phys. Rev. C68 (2003) 061902.

[11] S. Grigoryan and A. De Falco, ALICE Internal Note, ALICE-INT-2008-016 (2008).

[12] W.M. Alberico, A. Beraudo, A. De Pace, A. Molinari, Phys. Rev. D 72 (2005) 114011.

[13] C.Y. Wong, Phys. Rev. C 72 (2005) 034906; Phys. Rev. C 76 (2007) 014902. 